\title{
Impact of adverse events on prescribing warfarin in patients with atrial fibrillation: matched pair analysis
}

Niteesh K Choudhry, Geoffrey M Anderson, Andreas Laupacis, Dennis Ross-Degnan, Sharon-Lise T Normand, Stephen B Soumerai

\begin{abstract}
Objectives To quantify the influence of physicians' experiences of adverse events in patients with atrial fibrillation who were taking warfarin.

Design Population based, matched pair before and after analysis.

Setting Database study in Ontario, Canada.

Participants The physicians of patients with atrial fibrillation admitted to hospital for adverse events (major haemorrhage while taking warfarin and thromboembolic strokes while not taking warfarin). Pairs of other patients with atrial fibrillation treated by the same physicians were selected.

Main outcome measures Odds of receiving warfarin by matched pairs of a given physician's patients (one treated after and one treated before the event) were compared, with adjustment for stroke and bleeding risk factors that might also influence warfarin use. The odds of prescriptions for angiotensin converting enzyme (ACE) inhibitor before and after the event was assessed as a neutral control.

Results For the 530 physicians who had a patient with an adverse bleeding event (exposure) and who treated other patients with atrial fibrillation during the 90 days before and the 90 days after the exposure, the odds of prescribing warfarin was $21 \%$ lower for patients after the exposure (adjusted odds ratio $0.79,95 \%$ confidence interval 0.62 to 1.00$)$. Greater reductions in warfarin prescribing were found in analyses with patients for whom more time had elapsed between the physician's exposure and the patient's treatment. There were no significant changes in warfarin prescribing after a physician had a patient who had a stroke while not on warfarin or in the prescribing of ACE inhibitors by physicians who had patients with either bleeding events or strokes.

Conclusions A physician's experience with bleeding events associated with warfarin can influence prescribing warfarin. Adverse events that are possibly associated with underuse of warfarin may not affect subsequent prescribing.
\end{abstract}

\section{Introduction}

Clinical trials have shown that long term anticoagulation reduces the risk of stroke associated with atrial fibrillation, ${ }^{1}$ but warfarin is taken by only $30-60 \%$ of appropriate patients. ${ }^{2-4}$ Because about $15 \%$ of all strokes are attributable to atrial fibrillation, ${ }^{5}$ the clinical and economic consequences of underprescription of warfarin are profound. ${ }^{6}$

Physicians' overestimation of the risks of anticoagulation is the most consistently cited explanation for the observed patterns of warfarin use. ${ }^{7}$ These perceptions may be influenced by physicians' experiences with warfarin use in their patients ${ }^{8}$; physicians whose patients have had adverse events from anticoagulation may be less likely to prescribe warfarin. ${ }^{10}$ Unfortunately, the one study that assessed this association had a small sample size and asked physicians about the quality of their experiences prescribing warfarin to patients with atrial fibrillation without further characterising the adverse events. ${ }^{10}$

Adverse events associated with an action (for instance, a major haemorrhage in a patient with atrial fibrillation who had been prescribed warfarin) may have more influence on a physician's practice than adverse events associated with inaction (for instance, not prescribing warfarin to a patient with atrial fibrillation who subsequently has a thromboembolic stroke). ${ }^{11}{ }^{12}$ Accordingly, we sought to quantify the influence of both types of events on warfarin use for patients with atrial fibrillation.

\section{Methods}

\section{Setting and design}

We assembled a retrospective cohort of patients aged $\geq 66$ with non-valvular non-transient atrial fibrillation who were living in the community. We linked large healthcare databases that have been used extensively in other population based studies. ${ }^{13} 14$

We included all patients admitted to hospital from 1 January 1994 to 31 March 2002 with a primary ("most responsible") diagnosis or major comorbid diagnosis of atrial fibrillation (ICD-9 (international classification of diseases, ninth revision) code 427.3) on the basis of Canadian Institutes of Health Information $(\mathrm{CIHI})$ records. We excluded patients for whom atrial fibrillation was a complication after admission, who had valvular heart disease (defined as having inpatient diagnoses of mitral stenosis, prosthetic heart valves, or mitral or aortic valve repair or replacement before their admission with atrial fibrillation), who were likely to have perioperative atrial fibrillation (defined as having coronary artery bypass surgery, pericardial surgery, or structural cardiac repair within 30 days before their atrial fibrillation admission), who had hyperthyroidism or thyrotoxicosis within the past 12 months (based on discharge abstracts and prescriptions for antithyroid medications), who died during admission or within 60 days after discharge, who were residents of chronic care facilities, or who did not have a valid health card number.

For patients with more than one eligible admission, we included data only from the first. 


\section{Identification of adverse events}

To identify patients who experienced severe bleeding events associated with warfarin we searched for patients in our cohort who were readmitted with an upper gastrointestinal bleed (ICD-9 codes 531, 532, 534, 578.0, 578.1, 578.9) ${ }^{15}$ or intracerebral haemorrhage (ICD-9 code 431) ${ }^{16}$ after their initial admission and who had received a prescription for warfarin during the 120 days before the admission for bleeding. If a patient had more than one bleeding event, we included data only from the first.

To identify patients with atrial fibrillation who had a thromboembolic stroke while not on warfarin, we searched for patients who were readmitted with ischaemic stroke (ICD-9 code 434 or 436) and who had not received a prescription for warfarin in the 120 days before this admission. If a patient had more than one stroke, we included data only from the first.

\section{Identification of physicians and creation of cohorts}

Using billing claims from the Ontario Health Insurance Plan database, we identified the physicians responsible for the care of patients who experienced adverse events. The "principal provider" was defined as the physician who submitted the greatest number of outpatient service claims for care related to cardiac diagnoses (that is, hypertension, ischaemic heart disease, pulmonary embolism, conduction defects and arrhythmias, congestive heart failure, valvular heart disease, arteriosclerosis and aneurysms, and other diseases of the heart and circulatory system) in the six months after a patient experienced an adverse event. The date of hospital discharge for this patient was considered as the physician's "exposure date." If a physician had more than one exposure to a bleeding or stroke event, we considered only the first of each type. If a physician had exposure to both a bleeding and a stroke event, we considered each separately.

Using these definitions of exposure, we created two main cohorts. Our first cohort consisted of all patients with atrial fibrillation cared for by the principal providers of patients with bleeding events associated with warfarin. We excluded the actual patients who had experienced the bleeding events (that is, the index cases) and classified all other patients as having an admission before or after their physician's exposure date. Our second cohort was created by repeating this procedure for all patients cared for by the principal providers of atrial fibrillation patients who had had a stroke while not on warfarin.

\section{Patients' comorbidity}

We identified comorbidities in patients by searching hospital discharge abstracts and physicians' claims data for the presence of relevant diagnostic codes for the five years up to and including their index admission date as well as drug claims for the year before this date. Age $>75$, previous ischaemic stroke, congestive heart failure, hypertension, diabetes, and coronary artery disease were identified as risk factors for stroke. We also identified previous upper gastrointestinal bleeding, lower gastrointestinal bleeding, intracerebral haemorrhage, renal disease, liver disease, dementia or cognitive impairment, and use of antiplatelet agents and non-steroidal anti-inflammatory agents as risk factors for bleeding. We determined whether a cardiologist was involved in their care by assessing whether a cardiologist had submitted a claim for that patient in the six months after the hospital discharge date.

\section{Statistical analyses}

We used a matched pair before and after design to evaluate the impact of adverse events associated with warfarin on a physician's subsequent prescribing of warfarin. We selected pairs of patients treated by each exposed physician, one patient before

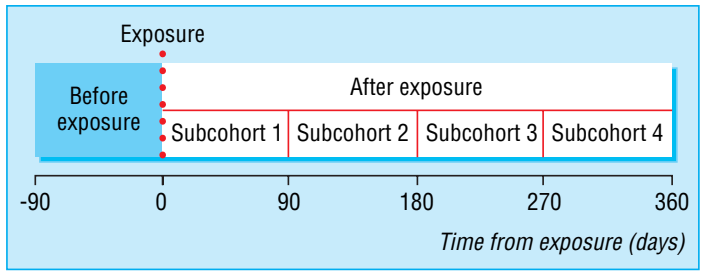

Strategy for analysis

and one patient after exposure, and compared their odds of warfarin receipt. Separate analyses were conducted for physicians who were exposed to bleeding and stroke events.

Our primary analysis compared warfarin use by the most recently admitted patient of a physician during the 90 days immediately before exposure with his or her first newly discharged patient during the 90 days after exposure. In subsequent analyses, we evaluated physicians who treated patients newly discharged in the same 90 days before exposure and the first newly discharged patient with atrial fibrillation in three other periods after exposure (91-180 days, 181-270 days, and 271-360 days, thus creating four subcohorts (figure).

By using this method, physicians served as their own controls, thereby reducing confounding due to fixed characteristics such as specialty training and practice style (for example, preferences for warfarin prescribing and tolerance of risk). We chose a 90 day exposure window to allow sufficient time for filling of prescriptions as patients covered by the Ontario Drug Benefit Program are dispensed a maximum of three months of medication.

To assess the specificity of our findings, we repeated our analyses using prescriptions for angiotensin converting enzyme (ACE) inhibitors in the same patients. If our results were attributable to adverse events associated with warfarin and not differences in patients' characteristics or changes in physicians' general tendencies to prescribe medications, the odds of ACE inhibitor prescribing should be the same for patients treated before and after exposure.

We compared the baseline characteristics of patients before and after exposure with paired $t$ tests and McNemar's tests. Odds ratios for the association between exposure to an adverse event and the likelihood of prescribing warfarin were estimated with univariate and multivariable conditional logistic regression. An odds ratio $<1$ indicates a reduced likelihood of prescribing warfarin after exposure. All analyses were performed with SAS version 8.2 (SAS Institute, Cary, NC).

\section{Results}

Of the 116200 patients with non-valvular non-transient atrial fibrillation identified during the study period, 3921 (3.4\%) were readmitted to hospital with an upper gastrointestinal $(\mathrm{n}=3478)$ or intracranial haemorrhage $(\mathrm{n}=443)$ while on anticoagulation. We identified the physician responsible for the care of 3120 $(79.6 \%)$ of these patients. Of these physicians, 530 treated other patients with atrial fibrillation in the 90 days before and the 90 days after the exposure. Table 1 shows the baseline characteristics from these 1060 patients. According to the guidelines of the American College of Chest Physicians (ACCP), ${ }^{17}$ 91.5\% of the patients before exposure and $92.1 \%$ of the patients after exposure group were at high risk of stroke associated with atrial fibrillation.

Patients treated in the 90 days after a physician's exposure to an adverse bleeding event were significantly less likely to receive 
Research

Table 1 Patients' characteristics by time period according to whether their physician had had a patient who had an adverse event related to warfarin use. Figures are numbers (percentages) of patients

\begin{tabular}{|c|c|c|c|c|c|c|}
\hline \multirow{2}{*}{ Characteristic } & \multicolumn{3}{|c|}{ Bleeding analysis* } & \multicolumn{3}{|c|}{ Stroke analysis* } \\
\hline & Before exposure $(\mathrm{n}=530)$ & After exposure $(n=530)$ & $P$ value & Before exposure $(n=704)$ & After exposure $(\mathrm{n}=704)$ & $P$ value \\
\hline Women & $262(49.4)$ & $245(46.2)$ & 0.29 & $344(48.9)$ & $334(47.4)$ & 0.58 \\
\hline Coronary artery disease & $219(41.3)$ & $223(42.1)$ & 0.80 & $320(45.5)$ & $283(40.2)$ & 0.04 \\
\hline Cardiology involvement & $185(34.9)$ & $176(33.2)$ & 0.43 & $202(28.7)$ & $203(28.8)$ & 0.94 \\
\hline \multicolumn{7}{|l|}{ Risk factors for stroke } \\
\hline Age $>75$ & $315(59.4)$ & $317(59.8)$ & 0.90 & $414(59.8)$ & $425(60.4)$ & 0.55 \\
\hline Previous stroke & $32(6.0)$ & $29(5.5)$ & 0.70 & $54(7.7)$ & $60(8.5)$ & 0.57 \\
\hline Congestive heart failure & $211(39.8)$ & $183(34.5)$ & 0.07 & $245(34.8)$ & $247(35.1)$ & 0.91 \\
\hline Hypertension & 397 (74.9) & $401(75.7)$ & 0.77 & $501(71.2)$ & $523(74.3)$ & 0.18 \\
\hline Diabetes & $134(25.3)$ & $124(23.4)$ & 0.47 & $161(22.9)$ & $144(20.5)$ & 0.26 \\
\hline \multicolumn{7}{|l|}{ Risk factors for bleeding } \\
\hline Previous upper Gl bleed & $35(6.6)$ & $42(7.9)$ & 0.42 & $45(6.4)$ & $36(5.1)$ & 0.30 \\
\hline Previous lower Gl bleed & $50(9.4)$ & $46(8.7)$ & 0.67 & $57(8.1)$ & $60(8.5)$ & 0.77 \\
\hline Previous intracerebral haemorrhage & $3(0.6)$ & $3(0.6)$ & 1.00 & $1(0.1)$ & $0(0.0)$ & 0.68 \\
\hline Liver disease & $4(0.8)$ & $3(0.6)$ & 0.71 & $7(1.0)$ & $0(0.0)$ & 0.02 \\
\hline Renal disease & $60(11.3)$ & $76(14.3)$ & 0.15 & $78(11.1)$ & 95 (13.5) & 0.17 \\
\hline Dementia & $11(2.1)$ & $6(1.1)$ & 0.23 & $12(1.7)$ & $9(1.3)$ & 0.49 \\
\hline NSAID use & $160(30.2)$ & $133(25.1)$ & 0.07 & $191(27.1)$ & $189(26.9)$ & 0.90 \\
\hline Anti-platelet use & $206(38.9)$ & $219(41.3)$ & 0.41 & $318(45.2)$ & $321(45.6)$ & 0.87 \\
\hline Warfarin use & $257(48.5)$ & 222 (41.9) & 0.03 & $260(36.9)$ & $253(35.9)$ & 0.70 \\
\hline ACE inhibitor use & $276(52.1)$ & $281(53.0)$ & 0.76 & $353(50.1)$ & $335(47.6)$ & 0.33 \\
\hline
\end{tabular}

$\mathrm{Gl}=$ gastrointestinal; NSAID=non-steroidal anti-inflammatory drug; ACE=angiotensin converting enzyme.

*Cohort before exposure consists of the last patients of exposed physicians during the 90 days immediately before exposure and the after exposure cohort consists of the first patients of exposed physicians during the 90 days after exposure.

†Based on paired $t$ tests and McNemar's tests.

a prescription for warfarin (odds ratio $0.77,95 \%$ confidence interval 0.61 to 0.98 ) than patients before the exposure. The results were unchanged after we adjusted for patients' covariates and involvement of a cardiologist in the care $(0.79,0.62$ to 1.00$)$ (table 2). Analyses based on other lengths of time after exposure yielded greater reductions in the odds of warfarin use (table 2). We found no significant association between exposure to bleeding events associated with warfarin and prescriptions for ACE inhibitors (table 2).

The cohort for our stroke analysis consisted of 8720 patients who had ischaemic strokes while not on warfarin. We were able to identify physicians for 6218 (71.3\%) of these patients, and 704 physicians treated patients in both the 90 days before and the 90 days after the exposure. Compared with patients treated before the exposure, patients treated after the exposure were less likely to have coronary artery disease $(\mathrm{P}=0.04)$ or liver disease $(\mathrm{P}=0.02)$ (table 1$)$. According to criteria from the American College of Chest Physicians, $92.2 \%$ of the patients before exposure

Table 2 Association between adverse events associated with warfarin and prescriptions for warfarin and ACE inhibitors in different comparison periods

\begin{tabular}{|c|c|c|c|}
\hline \multirow{2}{*}{$\begin{array}{l}\text { Comparison period } \\
\text { (days after exposure) }\end{array}$} & \multirow{2}{*}{$\begin{array}{c}\text { No of } \\
\text { physicians } \\
\text { evaluated }\end{array}$} & \multicolumn{2}{|c|}{ Odds ratio $(95 \% \mathrm{CI})$} \\
\hline & & Warfarin use* & ACE inhibitor use* \\
\hline \multicolumn{4}{|l|}{ Bleeding analysis } \\
\hline $0-90$ & 530 & 0.79 (0.62 to 1.00$)$ & $1.13(0.87$ to 1.47$)$ \\
\hline $91-180$ & 521 & 0.60 (0.46 to 0.79 ) & $1.16(0.90$ to 1.51$)$ \\
\hline $181-270$ & 488 & 0.61 (0.46 to 0.81 ) & 1.11 (0.84 to 1.46$)$ \\
\hline $271-360$ & 469 & 0.72 (0.54 to 0.97 ) & $1.06(0.79$ to 1.41$)$ \\
\hline \multicolumn{4}{|l|}{ Stroke analysis } \\
\hline $0-90$ & 704 & 0.95 (0.75 to 1.19$)$ & 0.88 (0.70 to 1.11$)$ \\
\hline $91-180$ & 664 & 1.05 (0.82 to 1.34$)$ & 0.99 (0.78 to 1.26$)$ \\
\hline $181-270$ & 656 & 1.22 (0.96 to 1.55$)$ & 1.17 (0.92 to 1.50$)$ \\
\hline $271-360$ & 621 & 1.23 (0.96 to 1.58$)$ & 1.08 (0.84 to 1.40$)$ \\
\hline
\end{tabular}

and $92.5 \%$ of the patients after exposure were at high risk of stroke associated with atrial fibrillation. All the patients (both from before and after exposure) had a similar likelihood of receiving warfarin (odds ratio $0.96,0.77$ to 1.19 ). Multivariable adjustment did not change the results nor did the use of different comparison periods (table 2). There was no association between exposure to ischaemic stroke in a patient with atrial fibrillation not on warfarin and use of ACE inhibitors (table 2).

\section{Discussion}

We studied the impact of adverse events associated with warfarin on prescribing in a population based cohort of patients with atrial fibrillation. Physicians were less likely to prescribe warfarin after one of their other patients had experienced a major adverse bleeding event associated with warfarin. Patients treated by physicians in the 90 days after the adverse event had a $21 \%$ reduced odds of receiving warfarin compared with patients treated by these same physicians before exposure. More strikingly, patients treated in the period 91-180 days after the adverse event had a $40 \%$ reduction in the odds of receiving warfarin compared with patients treated before the adverse event. This odds reduction, based on a baseline (before exposure) prescribing rate of $48 \%$, is equivalent to a $12 \%$ absolute and $26 \%$ relative decrease in the likelihood that a patient will receive warfarin. In contrast, a thromboembolic stroke in a patient with atrial fibrillation not on anticoagulation did not influence the odds that a physician will use warfarin in subsequent patients. As expected, the odds of ACE inhibitor prescribing were not influenced by a physician's exposure to either a bleeding or stroke event.

\section{Theoretical basis for the results}

These results provide empirical evidence for the existence of two frequently cited cognitive biases that affect clinical decisions. Firstly, Tversky and Kahneman's "availability heuristic" suggests that assessments of the probability of an event are influenced by the ease with which instances of the event can be recalled. ${ }^{18}$ 
Bleeding events related to anticoagulation, especially those that result in admission to hospital, are dramatic and easily remembered and, as we observed, seem to actually reduce warfarin prescribing. A similar logic has been proposed for patterns of use of other treatments including thrombolysis, ${ }^{19}$ antibiotics, ${ }^{20}$ and blood transfusions. ${ }^{21}$

Secondly, Feinstein's "chagrin factor" postulates that when choosing between alternatives, physicians avoid those actions that cause them the most regret. ${ }^{11}$ In the case of anticoagulation, physicians may have more chagrin associated with acts of commission (that is, adverse events associated with the administration of anticoagulation) than acts of omission (that is, adverse events associated with withholding anticoagulation), ${ }^{10}$ perhaps in keeping with the principle of non-maleficence or "do no harm."

\section{Limitations of the study}

There are several limitations to our study. Firstly, making causal inferences with administrative data is challenging. Our findings may have resulted from some other coincident event experienced by this group of physicians or differences between patients before and after exposure with respect to important but unmeasured factors. It is reassuring that our results were unchanged when we adjusted for well accepted clinical variables. Moreover, differences in cardiac risk profiles of patients before and after exposure would probably have been reflected in changes in ACE inhibitor prescribing, but this was not observed.

Secondly, our results may not be generalisable to all physicians who treat patients with atrial fibrillation. We included physicians in our analysis if they had had a patient who had experienced a bleeding event associated with warfarin, and these physicians at baseline are most likely to prescribe warfarin. The difference in the rates of warfarin prescribing before exposure in our two sets of analyses (48.5\% in the bleeding analyses and $36.9 \%$ in the stroke analyses) supports this assertion. Moreover, compared with physicians who were not included in our analysis, physicians in our bleeding cohort were significantly more likely to be cardiologists and to treat more patients with atrial fibrillation-both would be expected to be associated with higher rates of warfarin use. ${ }^{22}$

Thirdly, the relationship between physicians and patients is not directly identifiable within our data and we assigned physicians to patients based on service claims for cardiac related diagnoses. These physicians may not have been aware of the bleeding event and stroke events, especially when they were making prescribing decisions for other patients they treated shortly thereafter. However, this would reduce the likelihood of finding a reduction in warfarin prescribing after an adverse bleeding event; our results may therefore underestimate the true effect of adverse experiences on warfarin prescribing.

Finally, our analysis of the impact of ischaemic stroke on warfarin prescribing may have been underpowered to detect small effects. With our sample of 1408 patients (704 matched pairs), we had $80 \%$ power to detect a $30 \%$ increase in the odds of warfarin prescribing. A much larger study would have been required to detect a smaller effect (for example, 5000 patients for a $15 \%$ increased odds) should such an effect really exist.

\section{Implications and conclusions}

To our knowledge, this is the first study to use a population based dataset to assess the impact of specific dramatic adverse clinical events on subsequent patterns of care. Given the inherent limitations of prospective assessment methods, including the biases induced by directly questioning physicians, ${ }^{23}$ this is a potentially powerful tool for understanding clinical behaviour.

\section{What is already known on this topic}

Warfarin is underprescribed to patients with atrial fibrillation

Physicians' overestimation of the risks of anticoagulation is a commonly cited explanation for the observed patterns of warfarin use

These perceptions may be influenced by physicians' experiences with warfarin use in their patients

\section{What this study adds}

Physicians are less likely to prescribe warfarin after one of their patients has a major adverse bleeding event associated with warfarin

A thromboembolic stroke in a patient with atrial fibrillation not on anticoagulation does not influence the odds that a physician will use warfarin in subsequent patients.

Our findings provide further insight about reasons for underuse of warfarin in the treatment of atrial fibrillation and, more generally, about patterns of care for other similar conditions. As the prevalence of atrial fibrillation is increasing ${ }^{24}$ and ischaemic strokes related to atrial fibrillation are a burden for patients and the healthcare system, efforts to address specific barriers to appropriate atrial fibrillation care are essential. Based on our results, these interventions should also address physicians' perceptions of risk associated with warfarin use.

Contributors: All authors were responsible for concept and design and critical revision of the manuscript. NKC acquired, analysed, and interpreted data, drafted the manuscript, provided statistical expertise, and is guarantor. GMA was responsible for administrative, technical, or material support and supervised the study. AL was responsible for administrative, technical, or material support. DR-D and SBS supervised the study. S-LTN provided statistical expertise and supervised the study.

Funding: Harvard Pilgrim Health Care Foundation and a Canadian Institute for Health Research Chronic Disease New Emerging Theme (NET) programme grant (NET 54010). NKC was also supported by the Harvard Medical School fellowship in pharmaceutical policy research, a Frank Knox scholarship from Harvard University, and a Canadian Institutes of Health Research postdoctoral fellowship. SBS and DR-D are investigators in the HMO Research Network Center for Education and Research in Therapeutics, funded by the US Agency for Healthcare Research and Quality, and were also supported by grant No R01 AG022362-01 from the National Institute on Aging. AL is a senior scientist of the Canadian Institutes of Health Research.

Competing interests: None declared.

Ethical approval: The ethics review board of Sunnybrook and Women's College Health Sciences Centre, Toronto, approved this study.

1 Hart RG, Benavente O, McBride R, Pearce LA. Antithrombotic therapy to prevent stroke in patients with atrial fibrillation: a meta-analysis. Ann Intern Med 1999;131:492501.

2 Fang MC, Stafford RS, Ruskin JN, Singer DE. National trends in antiarrhythmic and antithrombotic medication use in atrial fibrillation. Arch Intern Med 2004;164:55-60.

Cohen N, Almoznino-Sarafian D, Alon I, Gorelik O, Koopfer M, Chachashvily S, et al. Warfarin for stroke prevention still underused in atrial fibrillation: patterns of omission. Stroke 2000;31:1217-22.

4 Majeed A, Moser K, Carroll K. Trends in the prevalence and management of atrial fibrillation in general practice in England and Wales, 1994-1998: analysis of data from the general practice research database. Heart 2001;86:284-8.

Wolf PA, Abbott RD, Kannel WB. Atrial fibrillation: a major contributor to stroke in the elderly. The Framingham study. Arch Intern Med 1987;147:1561-4.

6 Stewart S, Murphy N, Walker A, McGuire A, McMurray JJV. Cost of an emerging epiStewart S, Murphy N, Walker A, McGuire A, McMurray JJ . Cost of an emerging e
demic: an economic analysis of atrial fibrillation in the UK. Heart 2004;90:286-92.

Bungard TJ, Ghali WA, Teo KK, McAlister FA, Tsuyuki RT. Why do patients with atrial

fibrillation not receive warfarin? Arch Intern Med 2000;160:41-6.
Freeman AC, Sweeney K. Why general practitioners do not implement evidence: qualitative study. BMJ 2001;323:1100-2. 
9 Gross CP, Vogel EW, Dhond AJ, Marple CB, Edwards RA, Hauch O, et al. Factors influencing physicians' reported use of anticoagulation therapy in nonvalvular atrial fibrillation: a cross-sectional survey. Clin Ther 2003;25:1750-64

10 Beyth RJ, Antani MR, Covinsky KE, Miller DG, Chren MM, Quinn LM, et al. Why isn't warfarin prescribed to patients with nonrheumatic atrial fibrillation? J Gen Intern Med 1996;11:721-8.

11 Feinstein AR. The "chagrin factor" and qualitative decision analysis. Arch Intern Med $1985 ; 145: 1257-9$

12 Monette J, Gurwitz JH, Rochon PA, Avorn J. Physician attitudes concerning warfarin for stroke prevention in atrial fibrillation: results of a survey of long-term care practitioners. J Am Geriatr Soc 1997;45:1060-5.

13 Juurlink DN, Mamdani M, Kopp A, Laupacis A, Redelmeier DA. Drug-drug nteractions among elderly patients hospitalized for drug toxicity. JAMA 2003;289:1652-8.

14 Bell CM, Redelmeier DA. Mortality among patients admitted to hospitals on weekends as compared with weekdays. N Engl J Med 2001;345:663-8.

15 Raiford DS, Perez Gutthann S, Garcia Rodriguez LA. Positive predictive value of ICD-9 codes in the identification of cases of complicated peptic ulcer disease in the Saskatchewan hospital automated database. Epidemiology 1996;7:101-4.

16 Tirschwell DL, Longstreth WT Jr. Validating administrative data in stroke research. Stroke 2002;33:2465-70

17 Singer DE, Albers GW, Dalen JE, Go AS, Halperin JL, Manning WJ. Antithrombotic therapy in atrial fibrillation: the seventh ACCP conference on antithrombotic and thrombolytic therapy. Chest 2004;126:429-56S.

18 Tversky A, Kahneman D. Judgement under uncertainty: heuristics and biases. Science 1974;185:1124-31.

19 Soumerai SB, McLaughlin TJ, Gurwitz JH, Guadagnoli E, Hauptman PJ, Borbas C, et al Effect of local medical opinion leaders on quality of care for acute myocardial infarction: a randomized controlled trial. JAMA 1998;279:1358-63.

20 Damoiseaux RA, de Melker RA, Ausems MJ, van Balen FA. Reasons for non-guidelinebased antibiotic prescriptions for acute otitis media in the Netherlands. Fam Pract $1999 ; 16: 50-3$
21 Salem-Schatz SR, Avorn J, Soumerai SB. Influence of clinical knowledge, organizational context, and practice style on transfusion decision making. Implications for practice change strategies. JAMA 1990;264:476-83.

22 Kellen JC, Russell ML. Physician specialty is associated with differences in warfarin use for atrial fibrillation. Can J Cardiol 1998; 14:365-8.

23 Adams AS, Soumerai SB, Lomas J, Ross-Degnan D. Evidence of self-report bias in assessing adherence to guidelines. Int J Qual Health Care 1999;11:187-92.

24 Go AS, Hylek EM, Phillips KA, Chang Y, Henault LE, Selby JV, et al. Prevalence of diagnosed atrial fibrillation in adults: national implications for rhythm management and stroke prevention: the anticoagulation and risk factors in atrial fibrillation (ATRIA) study. JAMA 2001;285:2370-5.

(Accepted 8 November 2005)

doi $10.1136 /$ bmj. 38698.709572 .55

Harvard Medical School and Brigham and Women's Hospital, Boston, USA 02120 Niteesh K Choudhry instructor in medicine

Department of Health Policy, Management and Evaluation, Faculty of Medicine, University of Toronto, Toronto, Canada

Geoffrey M Anderson chair in health management strategies

Institute of Clinical Evaluative Sciences, Toronto, Canada

Andreas Laupacis chief executive officer

Department of Ambulatory Care and Prevention, Harvard Medical School and

Harvard Pilgrim Health Care, Boston, USA

Dennis Ross-Degnan associate professor

Stephen B Soumerai professor

Department of Health Care Policy, Harvard Medical School, Boston, USA

Sharon-Lise T Normand professor of biostatistics

Correspondence to: N K Choudhry nchoudhry@partners.org 